\title{
All cellulose electrospun water purification membranes nanotextured using cellulose nanocrystals
}

\author{
Lee A. Goetz $\cdot$ Narges Naseri $\cdot$ Santhosh S. Nair $\cdot$ Zoheb Karim $•$ \\ Aji P. Mathew (1)
}

Received: 10 November 2017 / Accepted: 12 March 2018/Published online: 16 March 2018

(C) The Author(s) 2018

\begin{abstract}
Cellulose acetate (CA) fibers were electrospun on a mesh template to create specific surface and pore structures for membrane applications. The mesh template CA fiber mats were impregnated with cellulose nanocrystals at varying weight percentages. The membranes showed nanotextured surfaces and improved mechanical properties post impregnation. More importantly, the hydrophilicity of the original CA fibers was increased from a hydrophobic contact angle of $102^{\circ}-0^{\circ}$ thereby creating an anti-fouling membrane surface structure. The membranes showed rejection of $20-56 \%$ for particles of $0.5-2.0 \mu \mathrm{m}$, indicating potential of these membranes in rejecting microorganisms from water. Furthermore, high rejection of dyes (80-99\%) by adsorption and potential application as highly functional affinity membranes was demonstrated. These membranes can therefore be utilized as all-cellulose, green, scalable and low cost high flux membranes $(>20,000 \mathrm{LMH})$ for water
\end{abstract}

Electronic supplementary material The online version of this article (https://doi.org/10.1007/s10570-018-1751-1) contains supplementary material, which is available to authorized users.

L. A. Goetz · Z. Karim · A. P. Mathew

Division of Materials Science, Luleå University of

Technology, 97187 Luleå, Sweden

N. Naseri · S. S. Nair · A. P. Mathew $(\varangle)$

Department of Materials and Environmental Chemistry,

Stockholm University, 10691 Stockholm, Sweden

e-mail: aji.mathew@mmk.su.se cleaning applications in food industry where microorganisms and charged contaminants are to be removed.

Keywords Electrospinning - Cellulose nanocrystals · Water permeability · Size exclusion . Adsorption

\section{Introduction}

Membranes and membrane processes are now an integral part of the food processing chain; an estimated $20-30 \%$ of the membranes used in manufacturing industries worldwide are used by the food industry (Daufin et al. 2001; Inc 2004; Lipnizki 2010). In food industry biological contaminants is a major concern and fresh-cut produces include minimally processed products, which result in very high microbial loads, the initial microbiological contamination ranging from $10^{5}$ to $10^{7} \mathrm{CFU} / \mathrm{g}$ (Francis et al. 1999). The most significant are Listeria monocytogenes, which can grow in the cooling chain, and Enterobacteriaceae, like Salmonella enterica or Escherichia coli (De Oliveira et al. 2011). The European commission has identified that fresh-food microorganisms associated with outbreaks include bacteria, viruses, and parasites, and the World Health Organization reported in 2015 the first-ever estimates of disease burden caused by 31 foodborne agents (bacteria, viruses, 
parasites, toxins and chemicals) at global and regional levels (Directorate 2002; Shannon et al. 2008).

Water purification technology in food industry also faces some major technical barriers, especially while dealing with removal of small molecules and viruses and nanofiltration or reverse osmosis process is accompanied with high energy consumption and low water flux (Manth et al. 2003; Shahmansouri and Bellona 2015). Ultrafiltration membranes systems can be a solution as they are typically more compact, require lower pressure than reverse osmosis or nanofiltration membrane systems, and also have the ability to remove harmful biologics while maintaining a relatively high flux, though the removal of smaller molecules and ions can still be challenging. In addition, membrane fouling compromises the benefits of membranes due to reduced performance, reduced flux and thereby increase of cost (Flemming et al. 1997; Mohammad et al. 2012).

Food industry is a major water consuming sector and about $70 \%$ of the water consumption in these sectors is for cleaning and decontamination processes which calls for intelligent design of membrane operations aimed at reducing the dose of disinfectant agents and saving water by improving recyclability. A new generation of ultrafiltration membranes including biocidal functionalization with the aim of limiting biofouling and controlling the level of pathogens in process water is therefore needed. Reduction of water and energy inputs in the food chain provided by the development of nanotechnology advances and the use of novel approaches to control of water borne microorganisms would also result in a better microbiological quality of processed food and cleaning of water.

Membranes with charge have been successful in the recent years also due to better fouling resistance. The utilization of biological materials, such as cellulose and chitin, in membrane applications is a current research goal and its nanoscaled derivatives have shown promising applications in the membrane and water filtration as functional entities with high flux and high rejection driven by surface adsorption. Our studies have shown that nanocellulose in native form or with additional surface functionalization (TEMPO oxidation, enzymatic phosphorylation, cationization, etc.) have significant adsorption efficiency towards metal ions, nitrates, dyes, humic acid, etc. from industry effluents (Karim et al. 2014; Liu et al. 2015;
Ma et al. 2011a, b; Mautner et al. 2016; Sehaqui et al. 2015; Gopakumar et al. 2017) One challenge however is the processing of membrane with control of pore structure and layering of the different layers of the membrane without compromising on the membrane flux, functionality and rejection capacity and the green image of 'nanocellulose'.

Electrospinning, being an established process, is becoming a popular route in producing membranes with an open yet interconnected pore structure and high surface area (Gopal et al. 2006; Huang et al. 2003). Electrospun membranes are used for removal of micron scaled pollutants in water and there are several attempts to improve the functionality and stability of these membranes by addition of nanoparticles, blending with functional polymers, crosslinking, etc. (Huang et al. 2013; Ma et al. 2005; Qin et al. 2015). The electrospun fibrous membranes have also shown potential as an excellent support layer for a secondary active surface-by coating individual fiber or by creating a more complete secondary layer of a bilayered composite membrane (Ma et al. 2011a, b; Gopi et al. 2017) Ma et al. (2011b) have shown that TEMPO oxidized cellulose nanocrystals with negative functional groups can be used to functionalize electrospun membranes, which provides a route to reject bacteria via size exclusion and reject virus via adsorption. We have also shown that stand-alone and biobased electrospun membranes of cellulose acetate with sufficient mechanical properties and flux for membrane as well as anti-fouling performance can be obtained via impregnation using chitin nanocrystals (Goetz et al. 2016). While typically the electrospun mat is randomly aligned, there has also been recent interest in electrospinning fibers on to templates to create aligned electrospun mats. However, templated electrospun membranes have not been exploited for functionalization with nanocrysals and its use in water filtration.

This work seeks to demonstrate an effective way to create high flux-renewable membranes for water filtration with the adsorption functionality of biobased cellulose nanocrystals on the mesh-template electrospun cellulose acetate fiber mats. The work aims to exploit the potential of cellulose nanocrystals functionalized electrospun cellulose acetate applications to reject $0.05-2.0 \mu \mathrm{m}$ particles via size exclusion while providing additional functionality as adsorption, antifouling and improved mechanical stability. 


\section{Experimental section}

Materials

Cellulose acetate (CA), $M_{n}$ 50,000, was purchased from Sigma-Aldrich Chemistry, USA. Acetic acid $\left(96 \%\right.$, EMSURE $\left.^{\circledR}\right)$ and acetone, analysis grade, were purchased from Merck KGaA (Germany). Polystyrene latex microspheres were purchased from Alfa Aesar in $2.5 \mathrm{wt} \%$ suspension with particle sizes of $0.05,0.5$, and $2.5 \mu \mathrm{m}$, individually. All chemicals were used as received without further purification.

Cellulose nanocrystals (CNCs) were prepared following the process reported earlier by Mathew et al. (2014). Unbarked wood was hydrolyzed using dilute acid in a bioethanol pilot plant and refined by solvent extraction and bleaching to obtain pure cellulose. Cellulose, after bioethanol process in a plant was supplied by SP Processum, Örnsköldsvik, Sweden $(17 \mathrm{wt} \%)$. The purified cellulose from bioethanol process was made into $1.5 \mathrm{wt} \%$ suspensions, mixed by shear mixture and passed through the homogenizer, 10 times to obtain a thick gel of CNCs.

\section{Preparation}

Cellulose acetate, $5.0 \mathrm{~g}\left(\mathrm{M}_{\mathrm{n}}\right.$ 50,000), was dissolved in a $45 \mathrm{~g}$ (1:1) mixture of concentrated acetic acid and acetone and stirred overnight (12 h) to ensure complete dissolution (Inc 2004). The viscosity and conductivity of the cellulose acetate electrospinning solution were $1144 \mathrm{mPa} . \mathrm{s}$ and $8.67 \mathrm{mScm}^{-1}$, respectively. Electrospinning of the cellulose acetate solution was undertaken using the $150 \mathrm{~mm}$ Laboratory Electrospinning Platform, Electrospinz-ES1a, New Zealand, attached to a high voltage supplier, with the solution pumped through a $20 \mathrm{ml}$ plastic syringe, (BD Plasti-Pak syringe, USA), using a single syringe pump (Aladdin-1000, World Precision Instrument, USA). The cellulose acetate fibers were successfully electrospun on stainless steel wire mesh (150 mesh, Forsbergs Metallduk AB, Sweden), with a supplied voltage of $9 \mathrm{kV}, 150 \mathrm{~mm}$ tip to collector distance, and a flow rate of $10 \mathrm{ml} \mathrm{h}^{-1}$. Electrospinning was performed for $2 \mathrm{~h}$ at room temperature.

A schematic representation of the membrane processing is given in Fig. 1. The mesh template electrospun cellulose acetate mats were impregnated with the CNC suspensions via Buchner funnel filtration apparatus with the cellulose acetate membrane on a $90 \mathrm{~mm}$ diameter glass frit. Varying weight percentages of crystals were filtered through the electrospun mesh mats (Fig. 1a) and are described in Table (in Fig. 1). The mesh side was the top surface during impregnation.

\section{Characterization}

Atomic Force Microscopy (AFM) AFM images of $\mathrm{CNC}$ shows typical cellulose nanocrystal structure and the diameters were found to be in the range of 5-10 $\mathrm{nm}$ but are longer compared to typical wood based CNCs. The resulting nanocrystal impregnated cellulose acetate membranes were air dried for $24 \mathrm{~h}$ and then heated to $100{ }^{\circ} \mathrm{C}$ for $10 \mathrm{~min}$ to ensure binding between the cellulose and the cellulose acetate fibers (Fig. 1b). Membranes were weighed on an analytical balance before and after impregnation to determine actual mass of the nanocrystals on the electrospun cellulose acetate mat.

Scanning Electron Microscopy (SEM) The surface morphology of the electrospun fibers and the membrane were examined using a FEG-SEM (Zeiss, Merlin). The fiber samples were placed on conductive tape and sputter coated with tungsten. Images were taken operating at $2.5 \mathrm{kV}$ and $8 \mathrm{~mm}$ working distance. The retention of the polystyrene latex microspheres was imaged using SEM (TVM SEM), imaged at $15 \mathrm{kV}$ and about $20 \mathrm{~mm}$ working distance.

Flux and Permeability Flux and permeability were determined using a dead-end cell (HP 4750, Sterlitech, USA) with $\mathrm{N}_{2}$ gas to maintain constant pressure. The time for $300 \mathrm{ml}$ of distilled water to pass through the membranes was recorded and used for the flux calculations. Flux, $J$, was calculated as

$J=\frac{Q_{p}}{A_{m}}$

where $Q_{p}$ is the filtrate volume through the membrane per time and $A_{m}$ is the area of the membrane. $A_{m}$ $\left(14.6 \mathrm{~cm}^{2}\right)$ is a constant value provided by Sterlitech. Permeability was calculated as flux $(J)$ per pressure (bar) multiplied by membrane thickness $(\mathrm{mm})$.

Tensile Properties Tensile tests were performed using a universal testing machine, Shimadzu Autograph AG-X (Japan), with a load cell of $500 \mathrm{~N}$. Test specimens $(50 \mathrm{~mm} \times 5 \mathrm{~mm})$ were conditioned at $45 \%$ relative humidity for 1 week and were mounted 

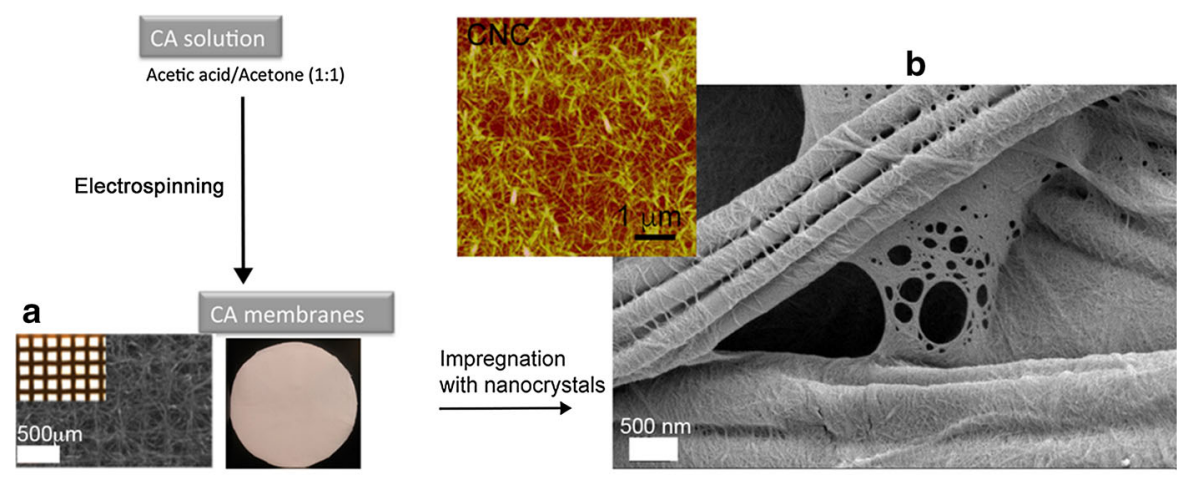

\begin{tabular}{cccc}
\hline Sample & CA (wt \%) & CNC (wt\%) & Coding \\
\hline 1 & 100 & 0 & CA \\
\hline 2 & 97 & 3 & CA-3CNC \\
3 & 92 & 8 & CA-8CNC \\
4 & 63 & 37 & CA-37CNC \\
5 & 55 & 45 & CA-45CNC \\
\hline
\end{tabular}

Fig. 1 Scheme showing the processing of CA membranes via electrospinning. a Mesh collector used for collecting fibers; the SEM images of corresponding membranes showing the mesh pattern and the photograph of the membrane. b AFM image of

on paper windows for ease of handling and mounting. A preload of $0.1 \mathrm{~N}$ was applied and a strain rate of $2 \mathrm{~mm} / \mathrm{min}$ and gauge length of $20 \mathrm{~mm}$ were used. The stress-strain curves were plotted from the measured load and sample extension (measured by video camera).

The stress is defined as

$\sigma=\frac{F}{A_{0}}$

and the strain as

$\varepsilon=\ln \left(\frac{L}{L_{0}}\right)$

where $F$ is the force at break, $A_{o}$ is the area of crosssection of the tensile sample, and $L_{0}$ is the initial sample length and $L$ is the sample length at break. The elastic modulus was calculated from the initial part of the slope from the stress-strain curve. 4-6 test samples were tested for each material and the average values are reported.

Contact Angle Equilibrium contact angles of the membrane surfaces against deionized water were measured in triplicate using a Kruss EasyDrop DSA1 (Germany) system. The surface energy was calculated using the Neumann equation of states the cellulose nanocrystals (CNCs) and the SEM image of the membranes showing the CNC coated electrospun fibers. Table showing the composition of the prepared materials and the codes which are used for each sample

approach (Moy and Neumann 1987; Li and Neumann 1992).

Microfiltration Performance by Particle Retention The retention performance of the membranes using polystyrene latex microspheres was evaluated by passing $20 \mathrm{ml}$ of the $0.05,0.5$, and $2.5 \mu \mathrm{m}$ polybead suspensions (Alfa Aesar, USA) through the membranes at an applied $\mathrm{N}_{2}$ gas pressure of 0.2 bar. Particle suspension concentrations were analyzed using UV-Vis spectrometer (PerkinElmer, Lambda 2S, USA).

Membrane Performance by Dye Adsorption The dye adsorption was carried out in static mode by treating the membranes $(0.05 \mathrm{~g})$ with $50 \mathrm{ml}$ of Victoria Blue $2 \mathrm{~B}$ with three different concentrations such as 10,25 , and $50 \mathrm{mg} / \mathrm{L}$, respectively. The dye adsorption on membranes of CA, CA-37CNC, and CA-45CNC were continuously monitored at different time intervals and quantitatively determined by UVVis spectrometer (PerkinElmer, Lambda 2S, Sweden). The absorbance of Victoria Blue 2B was calculated by Beer Lambert's law.

The amount of dye adsorbed was calculated using the formula as given below;

$\left.q=\left(C_{0}-C_{t}\right) V_{/ W}\right)$ 
where $C_{0}(\mathrm{mg} / \mathrm{L})$ is the initial dye concentration. $C_{t}$ is the dye concentration determined at the given time using UV-Vis spectrometer at the $\lambda$ max of $616 \mathrm{~nm}$. $V(\mathrm{~L})$ is the volume of the solution and $W(\mathrm{mg})$ is the weight of the adsorbent membrane in the solution.

The adsorption experiments were also conducted in dynamic mode using model water containing Victoria Blue 2B with an initial concentration of $2 \mathrm{mg} / \mathrm{L}$. Membranes were cut into circular discs with the diameter of $30 \mathrm{~mm}$ and used for experiments in crossflow mode at an applied $\mathrm{N}_{2}$ gas pressure of 0.2 bar using the dead-end cell (HP 4750, Sterlitech, USA). During the experiment $100 \mathrm{ml}$ of the dye solution was passed 10 times through the membranes. The solution after passing through the membrane is collected and the absorbance of the dye was recorded using UV-Vis spectrometer at the $\lambda$ max of $616 \mathrm{~nm}$. The percentage removal of dye was calculated using the formula as given below;

$\%$ Absorbance $=(A o-A t / A o) \times 100$

where $A o$ is the absorbance of dye solution before passing through the membrane and At is absorbance of dye solution after passing through the membranes.

Membrane Biodegradability Cellulose acetate membranes and the membranes coated with CNCs as functional layer before and after adsorption with dye (Victoria Blue 2B) and metal ions $\left(\mathrm{Cu}^{+2}\right)$ were immersed in water with different $\mathrm{pH}(4.52,7$, and 9.51) and at different temperature $\left(5{ }^{\circ} \mathrm{C}\right.$, room temp., and $\left.55^{\circ} \mathrm{C}\right)$. Furthermore, the membranes before adsorption were dipped to water sample (real wastewater) spiked with a mixture of pesticides solution. In parallel, membranes were buried in garden soil and in situ degradation of the membranes in soil at different temperatures $\left(5^{\circ} \mathrm{C}\right.$, room temp., and $55^{\circ} \mathrm{C}$ ) was investigated up to 2 months.

The membranes were recovered from water or soil at different time intervals. The membranes were washed with deionized water and were completely dried in the oven at $50{ }^{\circ} \mathrm{C}$ for $24 \mathrm{~h}$ till a constant weight was obtained and then they were weighed. The weight loss was calculated with respect to initial weight of the membranes using the following equation;

Weight $\operatorname{loss}(\%)=\left[\frac{(W 0-W t)}{W 0}\right] \times 100$ where $W_{0}$ is the initial weight of the membrane and $W_{t}$ refers to the weight of membrane at time t.

\section{Results and discussion}

Morphology of cellulose acetate membranes

Cellulose acetate fibers were successfully electrospun on wire mesh to create a surface template for future impregnation studies. The CA fibers preferentially aligned along the mesh structure (Fig. 2a). As electrospinning continued, the fibers started to 'fill in' the open pore structure, first diagonally and finally to create a random mat. The fibers show a smooth surface with complete fiber formation (Fig. 2b). The influence of the wire mesh on the electrospinning where the fibers preferentially align vertically, horizontally and then cross at $45^{\circ}$ angles to the junction points is also visible in these images and can provide further opportunities for tailorable web formation. The electrospun fiber mesh mats varied in overall thickness from 0.059 to $0.211 \mathrm{~mm}$.

The surface morphology to elucidate the methodology of the impregnation and subsequent formation of membrane mats was investigated using SEM. In all of the membranes, the cellulose nanocrystals formed web-like structures between the fibers-especially at the junction points. It was also noted that the grid patterns from the wire meshes are partially or completely lost after the impregnation with the nanocrystals.

The fibrous networks of the electrospun cellulose acetate fibers are visible after impregnation at the lower weight percentages of the cellulose nanocrystals (CA-3CNC, Fig. 2c). In comparison, the CA-45CNC demonstrates a different surface morphology whereby the cellulose nanocrystals ultimately form a dense top layer on top of the electrospun mat (Fig. 2d). When the underside of the membrane was imaged (Fig. 2e), larger webs of the cellulose nanocrystals were shown to be forming among and entrapping the CA fibers.

The density and porosity data given in supplementary data (Table S1) shows that density of the membrane increases and the porosity decreases with increasing the concentration of nanocrystals. The CA membranes had a porosity of $83 \%$, which is the typical range of porosity shown by electrospun random mats. 

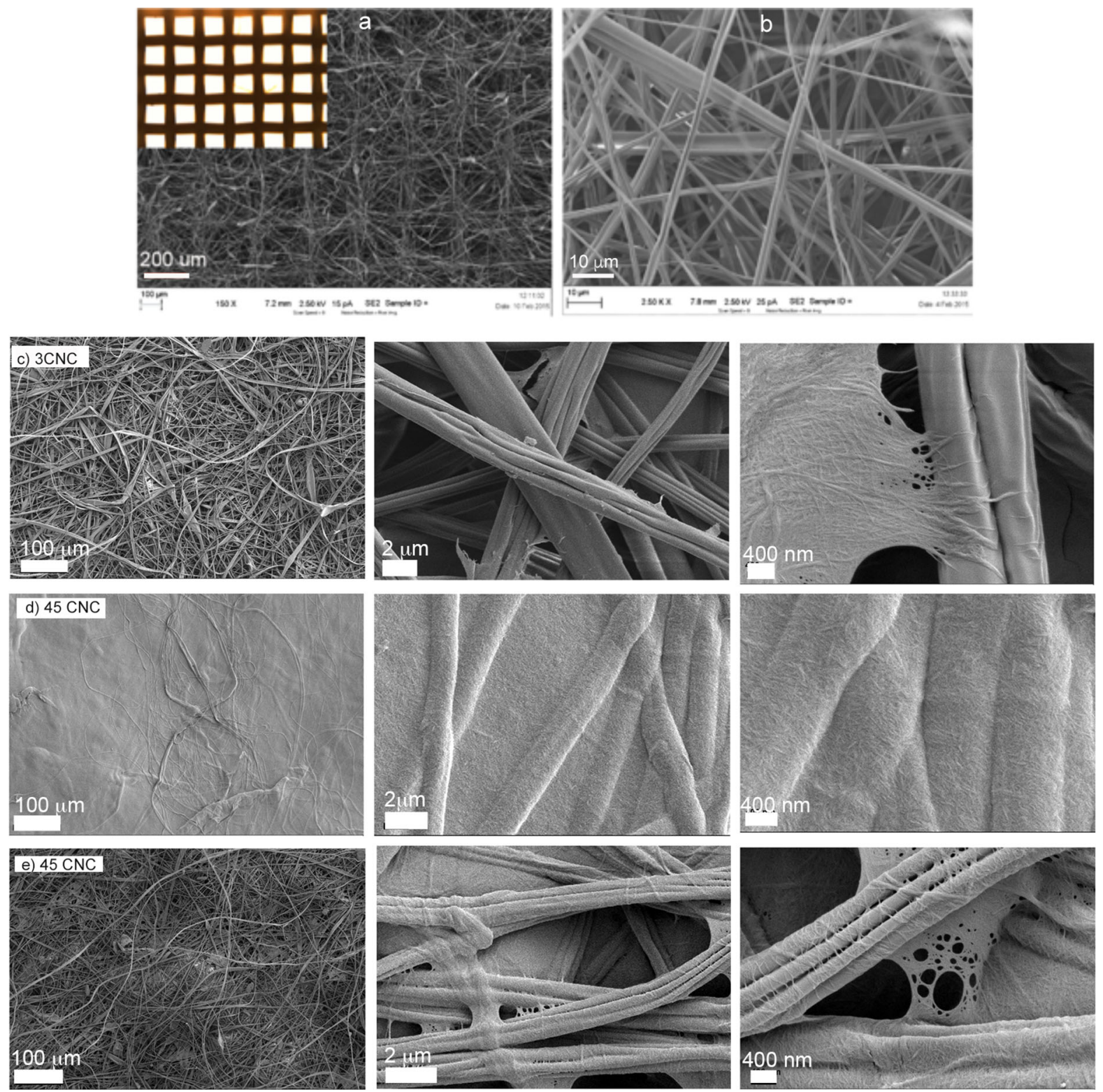

Fig. 2 SEM images of cellulose acetate fibers electrospun on to wire mesh screen $(\mathbf{a}, \mathbf{b})$ at different magnifications. SEM images of the CNC impregnated membranes showing the web formation, coating of the electrospun fibers with nanomaterials, c left to right; upper surface of CA-3CNC membrane at different

magnifications, d left to right; upper surface of CA-45CNC membranes at different magnification (a continuous nanocellulose layer on the electrospun mat is visible), e left to right; bottom surface of CA-45CNC membranes at different magnification

The lowest porosity (69\%) was observed for CA$45 \mathrm{CNC}$ content, as expected and is supported by the SEM images. The compaction of the membranes, with thickness decreasing from 143 microns for CA (measured after water washing and drying) to 52 microns for CA-45CNC support the decrease in

porosity and increase in density after CNC impregnation. The compaction in $\mathrm{CNC}$ impregnated $\mathrm{CA}$ membranes (increasing with CNC content) is not only due to the water washing, but also due to the $\mathrm{H}$-bonding formed between CNCs. 
Flux, permeability and wettability of membranes

The water flux through the membranes was studied to understand the effect of coating on the permeability and the data is given in Fig. 3. Figure 3 shows that the water flux increases with pressure in all cases, as expected. However, the trend line shows that slope increases for the functionalized membranes, indicating that water transport through the membranes is favored after coating with cellulose nanocrystals, in spite of the decreased pore diameters.

The water flux values are in the range of 5000-22,000 $\mathrm{Lm}^{-2} \mathrm{~h}^{-1}$ bar $^{-1}$ at $0.5-2$ bars for CA and $\mathrm{CA}$ functionalized with $3 \mathrm{wt} \%$ of cellulose nanocrystals. However, for high CNC content (45\%) the flux was $500-5000 \mathrm{Lm}^{-2} \mathrm{~h}^{-1} \mathrm{bar}^{-1}$ and was attained only at a high pressure of 6-8 bars. The surface morphology of these membranes explains the flux behavior, especially for CA-45CNC, which had drastically lower flux and permeability. Our earlier report on a similar system of CA membranes impregnated with $5 \mathrm{wt} \%$ chitin nanocrystals (ChNCs) also showed a high flux of $18,000 \mathrm{Lm}^{-2} \mathrm{~h}^{-1} \mathrm{bar}^{-1}$. In comparison, Ma et al. have reported a flux of $5900 \mathrm{Lm}^{-2} \mathrm{~h}^{-1}$ bar $^{-1}$ for PAN nanofiber mats with a support layer impregnated with cellulose

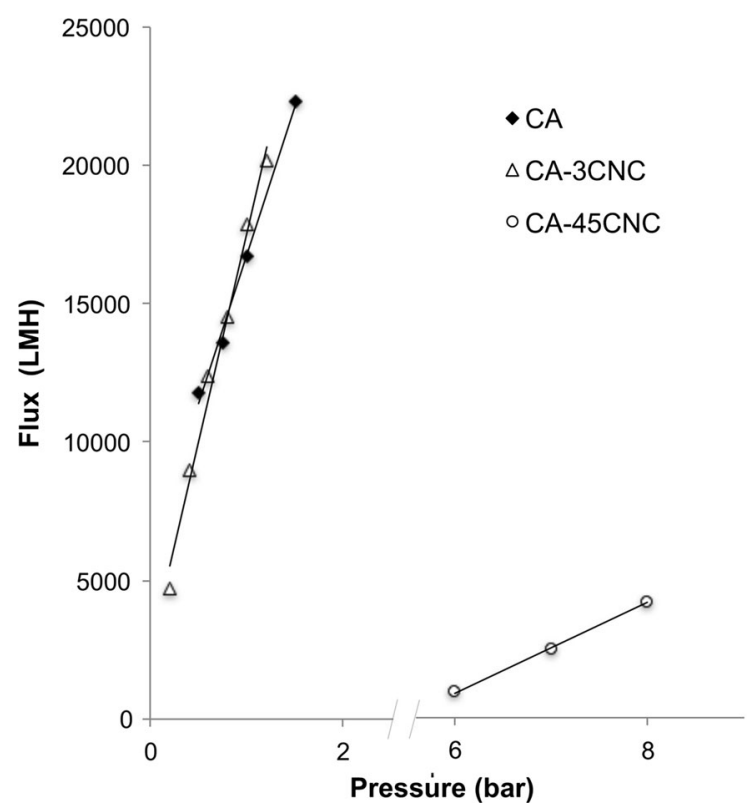

Fig. 3 The water flux through the CA and the nanocrystal functionalized membranes as a function of pressure nanocrystals and is lower than our current membrane (Ma et al. 2011b).

The flux and permeability through the membranes at 1 bar are given in supplementary data (Table S1). The flux increases slightly after impregnation with nanocrystals. However, permeability systematically decreased as a function of nanocrystal loading which is a direct function of pore structure and porosity; SEM images show a closing of pore structure as well as decrease in porosity with increased nanocrystal content.

The pore structure of the membranes with $3 \mathrm{wt} \%$ nanocrystal loading and absence of a support layer is considered favorable for high flux. Another possible explanation for the high flux is the increased hydrophilicity of the cellulose acetate membranes coated with nanocrystals confirmed by the contact angle measurements (Table 1).

The cellulose acetate membrane had a hydrophobic contact angle of $101.9^{\circ}$ while the CA-3CNC membranes demonstrated extreme hydrophilicity with a measured contact angle of $0^{\circ}$. In the case of CA$45 \mathrm{CNC}$ relatively higher contact angle of $50.3^{\circ}$ was observed and is probably due to the tighter CNC network that reduced the porosity. It is well documented that surface hydrophilicity can change the membrane flux as well as membrane anti-fouling performance (Ding et al. 2016; Kaur et al. 2007; Luo et al. 2005) and we have confirmed this in our earlier study where $\mathrm{ChNC}$ were coated on CA membranes (Goetz et al. 2016).

The impact of CNC on the surface roughness and antifouling of the membranes is not evaluated in this study though the SEM images clearly show that the impregnation with $\mathrm{CNC}$ increased the surface roughness; e.g. surface of the fibers (CA-3CNC) and are not as smooth as CA fibers. However, increasing the $\mathrm{CNC}$ loading (CA-45CNC) decreased the surface roughness due to formation of a compact film of $\mathrm{CNC}$ on the surface.

The work of adhesion and surface energy calculations also shows that there is an increase in surface activity after coating with the nanocrystals compared to pure CA membranes. Thus, surface functionalization with biobased nanocrystals can be a potential route to convert hydrophobic membrane surfaces into superhydrophilic and more interactive surfaces. 
Table 1 Contact angle data and surface characteristic of the CA membranes before and after nanocrystal functionalization

\begin{tabular}{|c|c|c|c|c|}
\hline & Contact angle $\left(^{\circ}\right)$ & Image & Work of adhesion $(\mathrm{mN} / \mathrm{m})$ & Surface energy $(\mathrm{mN} / \mathrm{m})$ \\
\hline $\mathrm{CA}$ & 101.9 & & 53.7 & 20.45 \\
\hline $\mathrm{CA}-3 \mathrm{CNC}$ & 0 & & 145.6 & $>70(72.3)$ \\
\hline CA-45CNC & 50.3 & & 119.3 & 53.76 \\
\hline
\end{tabular}

Mechanical properties

Figure 4 shows the mechanical properties of the membranes with and without the nanocrystal coating. At $3 \mathrm{wt} \% \mathrm{CNC}$ (CA-3CNC), the tensile strength of $\mathrm{CA}$ membranes remained unchanged but at high nanocrystal concentration the strength and modulus increased drastically and can be attributed to the stiffening effect of the nanocrystals coated on

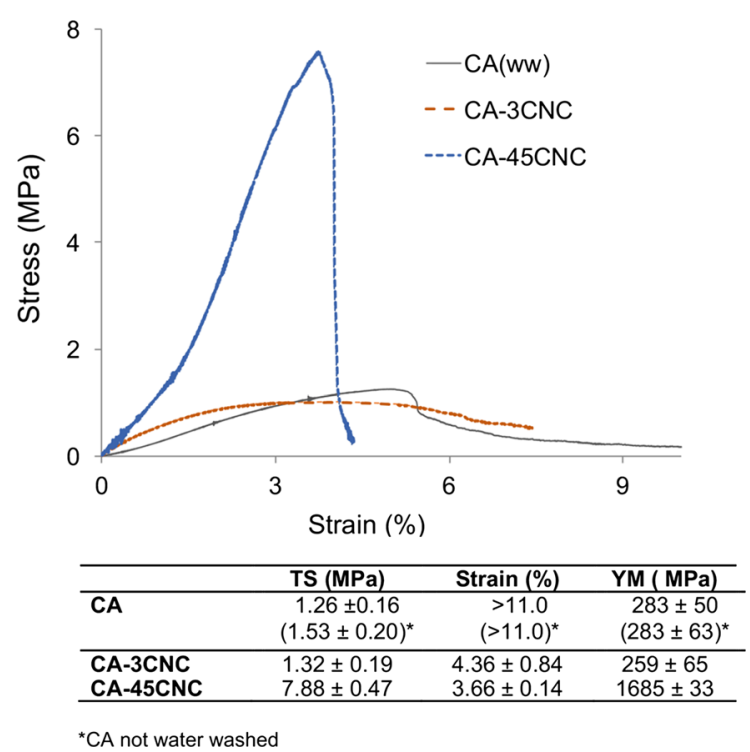

Fig. 4 Stress-strain curves and the mechanical property data of the CA membranes with cellulose nanocrystal functional coating of the electrospun fibers individual electrospun fibers as well as the closing of the pores (as evidenced by SEM images).

It is also worth mentioning that preparation of CA membranes with high loading of nanocrystals are usually highly challenging due to processibility issues and aggregation of nanoparticles at concentrations higher than $1 \mathrm{wt} \%$ (Herrera et al. 2011) and the surface coating with nanocrystals thereby provides a more viable and easy route to improve the mechanical properties of CA random mats. We have reported recently fully biobased membranes using micro to nanoscaled cellulose, where a thin layer of nanocellulose allows high flux and functionality (Karim et al. 2016). The study showed that CNC based functional layer increased the mechanical strength from 9 to $19 \mathrm{MPa}$, but decreased to $0.2 \mathrm{MPa}$ in wet conditions. Such drastic decrease is not expected for the current materials as CA electrospun mats are less susceptible to water compared to native cellulose microfibers. Therefore, the current approach where CA electrospun mats are surface coated with biobased nanocrystals can be a more efficient route to obtain all cellulose functional membranes that are stable in wet conditions.

Membrane performance

Adsorption of dyes To understand the functionality of the membranes and interaction with water contaminants, aqueous solutions of positively charged dye (Victoria Blue 2B) was treated with the membranes. 
The solution was evaluated at defined time intervals using a UV-Vis spectrometer. For $10 \mathrm{mg} / \mathrm{L}$ of dye concentration, it was found that CA, CA-37CNC, and CA-45CNC adsorbed 1.9, 87.5, and 99.9\%, respectively within $24 \mathrm{~h}$. It should be noted that $82.6 \%$ dye was adsorbed in $1 \mathrm{~h}$ for $\mathrm{CA}-45 \mathrm{CNC}$; however in the case of CA-37CNC the adsoprtion was $36.4 \%$ (Fig. 5a). Adsorption on pristine CA adsorption, in comparison, was very random, $(13.5 \%$ in $1 \mathrm{~h})$ and it was observed that the dyes were getting adsorbed and desorbed at various time intervals due to the lack of
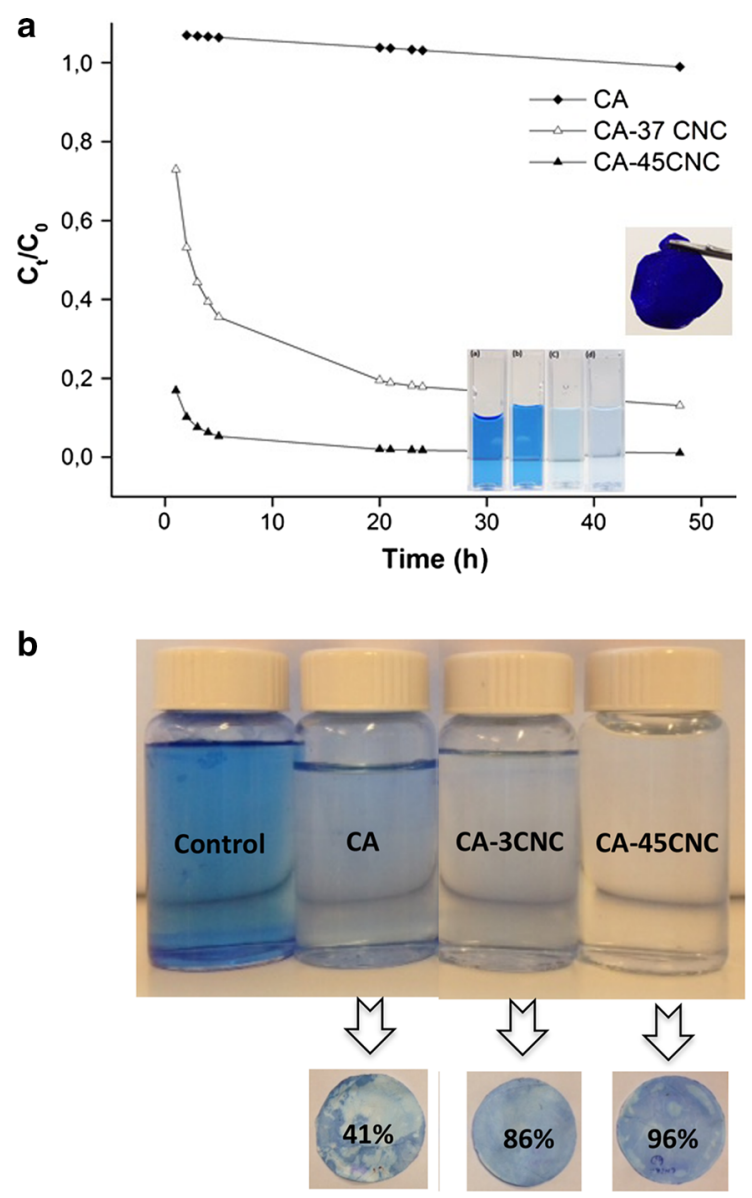

Fig. 5 The contaminant removal capability of the membranes via $\mathbf{a}$ static adsorption and $\mathbf{b}$ cross-flow filtration. a Performance of dye adsorption for concentration of $10 \mathrm{mg} / \mathrm{L}$; a initial dye color, the color after $24 \mathrm{~h}$ adsorption with membranes b CA, c CA-37CNC, and d CA-45CNC. The inset shows the image of CA-45CNC after $48 \mathrm{~h}$ adsorption. b Photographs showing the removal of dyes by cross-flow filtration for $10 \mathrm{~min}$ using $\mathrm{CA}$ electrospun membranes and the CNC coated membranes. The membranes after the filtration and the percentage removal of dyes are given electrostatic interactions. The dye adsorption for all the three membranes at concentration of $10 \mathrm{mg} / \mathrm{L}$ were tabulated and results are shown in Fig. 5a. The color degradation from the initial to final $(24 \mathrm{~h})$ was shown as photograph inset in Fig. 5a.

For higher dye concentrations such as 25 and $50 \mathrm{mg} / \mathrm{L}, \mathrm{CA}-45 \mathrm{CNC}$ was active and removed 84.36 and $52.62 \%$, respectively. In the case of CA-45CNC, a combination of adsorption and size exclusion can be expected due to the compact nature of the CNC coating. In the case of $25 \mathrm{mg} / \mathrm{L}$, adsorption improved by increasing the contact time by $24 \mathrm{~h}$, however, for $50 \mathrm{mg} / \mathrm{L}$, the adsorption reached saturation. The adsorption results are given in supplementary data (Table S2). The increase of CNC content to $45 \mathrm{wt} \%$ not only increased the rejection rate but also improved the mechanical robustness of the membrane. Figure $5 \mathrm{a}$ inset shows the CA-45CNC after the $48 \mathrm{~h}$ treatment with the dye solution.

To understand the effect of cross-flow filtration on dye removal, water containing positively charged dye was filtered through the membranes at a pressure of 0.2 bar. The removal of dyes after passing through the membranes was visually observable from the decreased intensity of blue color in the dye solutions (Fig. 5b). CA membranes without CNCs also removed only $41 \%$ of the dye whereas the removal capacity increased to $86 \%$ when $3 \mathrm{wt} \% \mathrm{CNC}$ was coated. The increase of $\mathrm{CNC}$ content to $45 \mathrm{wt} \%$ further increased the rejection to $96 \%$.

The mechanism of adsorption is the electrostatic interaction between the negatively charged $\mathrm{CNCs}$ and the positively charged dye irrespective of static or dynamic modes (Karim et al. 2014). In the case of CA$45 \mathrm{CNC}$ a combination of adsorption and size exclusion was expected due to the dense nature of the $\mathrm{CNC}$ coating.

Particle rejection The membrane performance via size exclusion was evaluated using particle analysis and the results are given in Table 2. It was concluded from the above results that a competition between the mechanical properties and the water flux exists and therefore CA membranes with $\mathrm{CNC}$ loadings between 3, 8, 37 and $45 \mathrm{wt} \% \mathrm{CNC}$ were used to study size exclusion phenomenon. CA membranes water washed and dried was used as control to account for the compaction effect. The CA membranes did not show significant rejection of 0.05 and $0.5 \mu \mathrm{m}$ particles and $23 \%$ rejection of $2 \mu \mathrm{m}$ particles. CNC impregnated 
Table 2 Particle rejection as a function of nanocrystal content

\begin{tabular}{lllll}
\hline Sample & Thickness $(\mathrm{mm})$ & $0.05 \mu \mathrm{m}, \%$ rejection & $0.5 \mu \mathrm{m}, \%$ rejection & $2.0 \mu \mathrm{m}, \%$ rejection \\
\hline CA & 0.143 & 4.5 & 6.45 & 22.71 \\
CA-3CNC & 0.116 & 5.91 & 24.62 & 25.86 \\
CA-8CNC & 0.092 & 3.16 & 8.75 & 17.93 \\
CA-37CNC & 0.059 & 2.5 & 20.25 & 20.43 \\
CA-45CNC & 0.052 & - & - & - \\
\hline
\end{tabular}

Fig. 6 The SEM studies showing the rejected PS particles with different sizes on the surface of the CA membranes with varying CNC contents

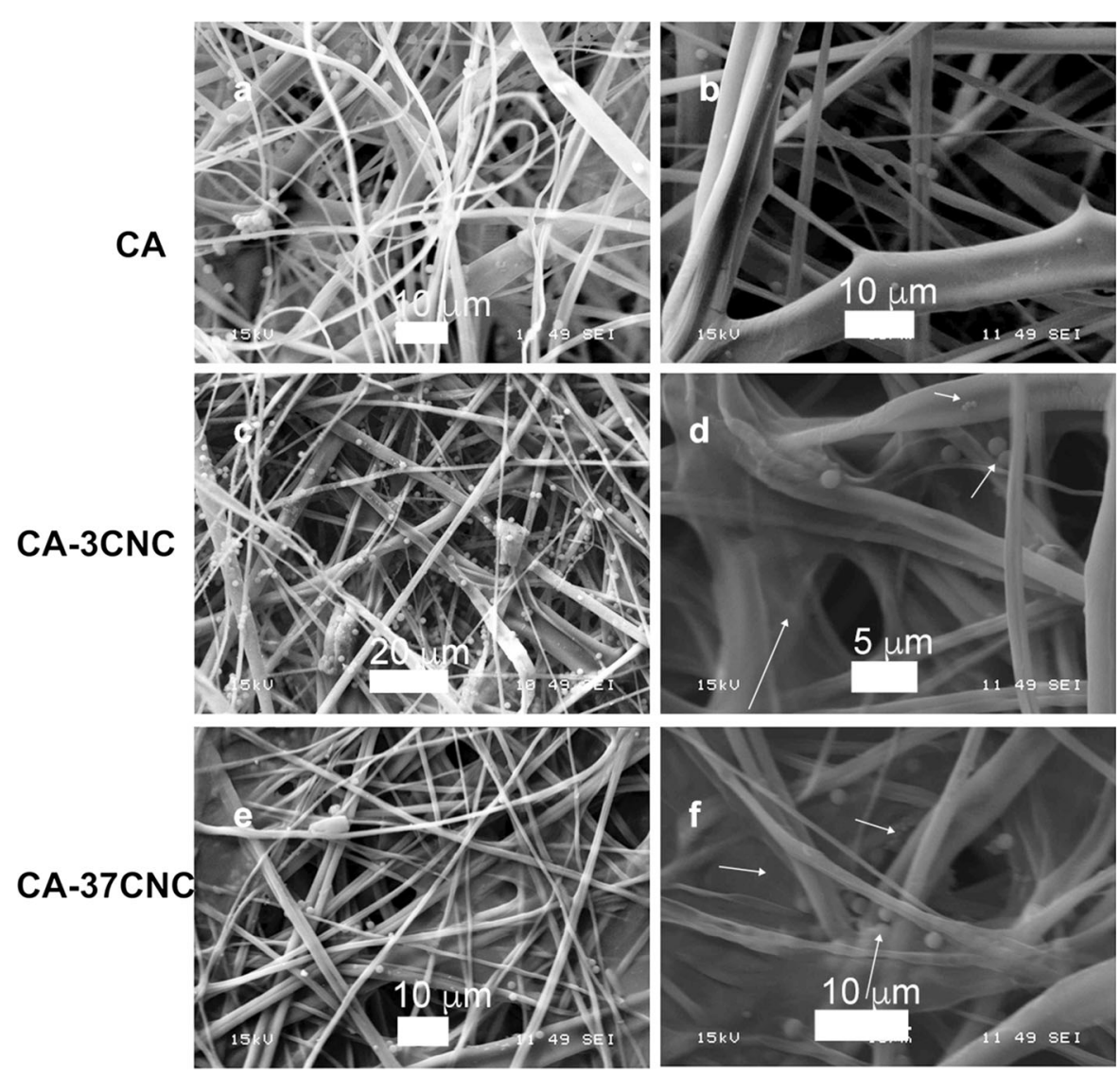

CA membranes showed the highest rejection of particles at $3 \mathrm{wt} \% \mathrm{CNC}$ content but decreased thereafter. A reversal of trend is noted at $37 \mathrm{wt} \%$ and is probably due to the fact that the decrease in membrane thickness is being compensated with a more continuous 'web' or 'film' formation of nanocrystals on the CA random mats. The $45 \% \mathrm{CNC}$ loaded membrane was not studied further due to lack of water flux at low pressure, which is expected due to the continuous film formation by $\mathrm{CNC}$ as observed in the SEM images shown in Fig. 2d.

Figure 6 shows the SEM images of the mats after the filtrations process. The images confirm the rejection of particles by all the three types of membranes. Also in the case of CA-3CNC and CA-37CNC the web or film of CNC layer (shown with arrows) on the CA electrospun fiber network is clearly visible after the 

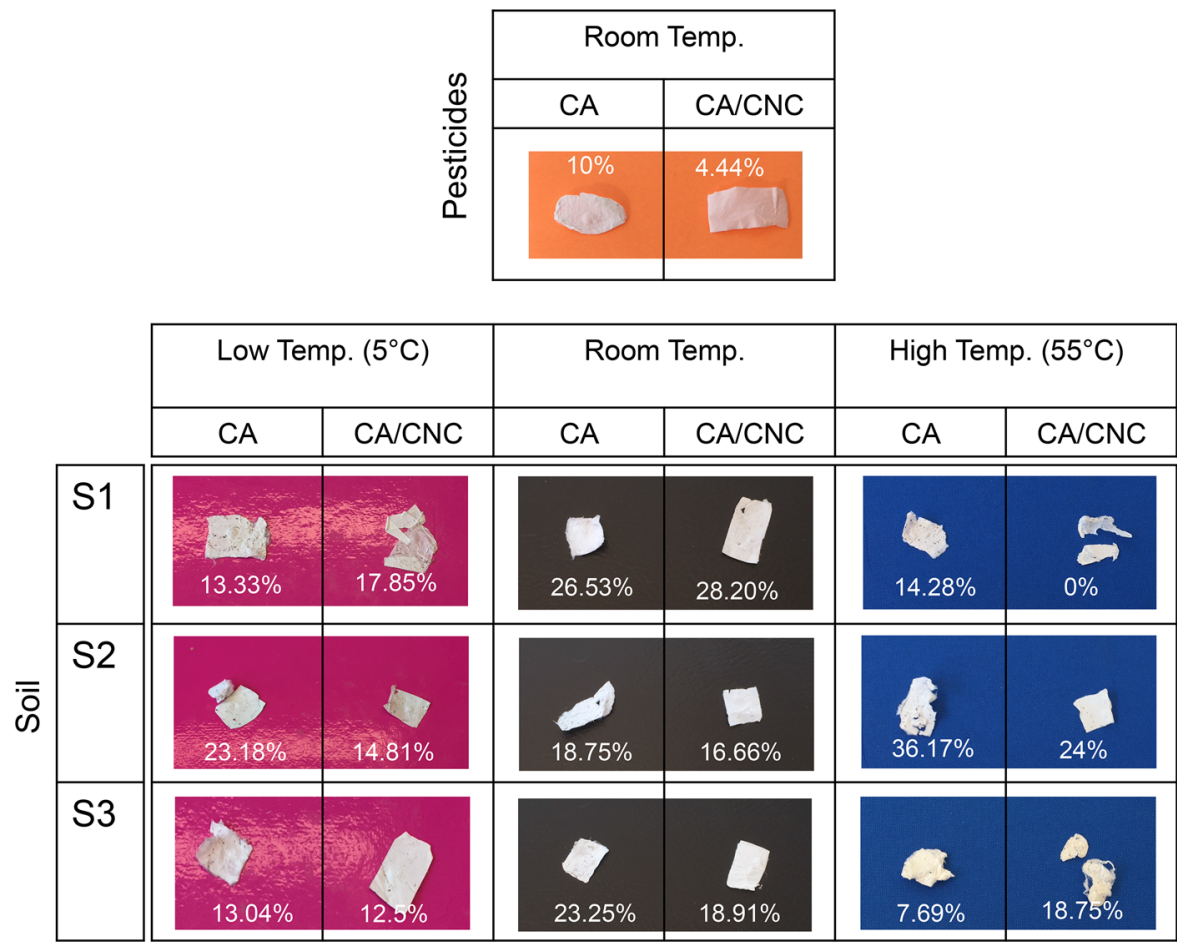
S1: without adsorption
S2: after adsorption with victoria blue
S3: after adsorption with $\mathrm{Cu}^{+2}$

Fig. 7 Biodegradation of the membranes after 2 months in real wastewater containing pesticides and in soil

filtration process, confirming the stability of the $\mathrm{CNC}$ layer in cross flow filtration.

The rejection of 0.5 and $2.0 \mu \mathrm{m}$ beads can be correlated to potential rejection of microorganisms as bacteria $(1-2 \mu \mathrm{m})$, yeast $(5-10 \mu \mathrm{m})$, protozoa $(50 \mu \mathrm{m})$ etc. via size exclusion during filtration. Owing to the scalability of CA based electrospun membranes and the advancements in $\mathrm{CNC}$ scaled up, the cost efficient commercial processing of all-cellulose electrospun membranes for water purification is viable.

Membrane biodegradability

Degradation of the membranes in water and soil was investigated up to two months and the visual appearances of membranes at different conditions are shown in Fig. 7.

In the case of degradation in water medium at different $\mathrm{pH}$ and temperatures as well as water sample (real wastewater) spiked with a mixture of pesticides solution, the membranes were mainly stable after 2 months and no degradation was observed regarding the visual appearance and weight loss. The water sample mixed with pesticides was not good enough for the growth of microorganism and after 2 months, the membranes showed good stability, especially after coating with CNCs. Thus, they are considered as potential candidates for water purification.

Soil burial tests for biodegradation of the membranes showed a different trend, at different temperatures and with different contaminant adsorption. Biodegradability of membranes in soil depends on the composition of soil (such as organic/inorganic matters and oxygen/carbon dioxide and water content), temperature and $\mathrm{pH}$. So, degradation rate and range can be changed in different region and season. In soil, the membranes were degraded more compared to the water medium (the values are shown in the inset) and it was also observed that higher temperature resulted in disintegration of the membranes and changes in the visual appearance after 2 months of incubation. 
However, no significant difference was noted in degradation of membranes before and after adsorption. Furthermore, the membranes did not show a systematic difference in degradation in soil due to CNC coating.

\section{Conclusions}

Cellulose nanocrystals were successfully infused on to the electrospun cellulose acetate fiber networks resulting in highly efficient surface treatment approach to prepare biobased membranes that combine size exclusion, adsorption and super hydrophilicity. The CA acetate mats spun on wire meshes showed grid patterns, which was considered beneficial in controlling the pore structure of the microfiber support layer. An hierarchical morphology is shown by the membranes where micron scaled electrospun fiber network is surface coated with nanocrystals, especially at lower nanocrystal loading, whereas a continuous web or coating and a concomitant increase in mechanical strength and modulus of the membranes as observed at higher CNC concentrations. The water flux through the membranes was very high $(22,000 \mathrm{LMH})$ and decreased when the nanocrystal network became more continuous at higher concentration. Moreover, the contact angle of the membranes dropped to $0^{\circ}$ after coating with nanocrystals indicating increased hydrophilicity and potential anti-fouling of these membranes towards hydrophobic entities. On the other hand, the high rejection of charged entities as dyes, attributable to adsorption confirms the application as affinity membranes. The pore structure was in microfiltration range and the networks showed moderate rejection of particles with $0.5-2$ microns. Biodegradation of the membranes was favorable for its application in water purification; showing limited degradation in polluted water and showing a tendency to degrade in soil for membranes with and without adsorbed contaminants as dyes and metal ions.

The study demonstrates an easy and successful route to functionalize and nanotexture electrospun fibers with CNCs. The resulting biobased membranes showed enhanced mechanical properties, high water flux, hydrophilicity/anti-fouling and pollutant removal capability via adsorption and size exclusion.
Acknowledgments The authors gratefully acknowledge financial support from FORMAS, under EU FP7, ERANET SUSFOOD funding, for CEREAL project; Dnr No. 222-201418.

Open Access This article is distributed under the terms of the Creative Commons Attribution 4.0 International License (http:// creativecommons.org/licenses/by/4.0/), which permits unrestricted use, distribution, and reproduction in any medium, provided you give appropriate credit to the original author(s) and the source, provide a link to the Creative Commons license, and indicate if changes were made.

\section{References}

Daufin G, Escudier J, Carrere H, Berot S, Fillaudeau L, Decloux M (2001) Recent and emerging applications of membrane processes in the food and dairy industry. Food Bioprod Process 79:89-102

De Oliveira MA, De Souza VM, Bergamini AMM, Martinis De, Pereira Elaine Cristina (2011) Microbiological quality of ready-to-eat minimally processed vegetables consumed in Brazil. Food Control 22:1400-1403

Ding Z, Zhong L, Wang X, Zhang L (2016) Effect of lignincellulose nanofibrils on the hydrophilicity and mechanical properties of polyethersulfone ultrafiltration membranes. High Perform Polym 28:1192-1200

Directorate C (2002) Risk profile on the microbiological contamination of fruits and vegetables eaten raw

Flemming H, Schaule G, Griebe T, Schmitt J, Tamachkiarowa A (1997) Biofouling-the Achilles heel of membrane processes. Desalination 113:215-225

Francis GA, Thomas C, O'beirne D (1999) The microbiological safety of minimally processed vegetables. Int J Food Sci Technol 34:1-22

Goetz LA, Jalvo B, Rosal R, Mathew AP (2016) Superhydrophilic anti-fouling electrospun cellulose acetate membranes coated with chitin nanocrystals for water filtration. J Membr Sci 510:238-248

Gopakumar DA, Pasquini D, Henrique MA, de Morais LC, Yves Grohens Y, Thomas S (2017) Meldrum's acid modified cellulose nanofiber-based polyvinylidene fluoride microfiltration membrane for dye water treatment and nanoparticle removal. ACS Sustain Chem Eng 5(2):2026-2033

Gopal R, Kaur S, Ma Z, Chan C, Ramakrishna S, Matsuura T (2006) Electrospun nanofibrous filtration membrane. J Membr Sci 281:581-586

Gopi S, Balakrishnan P, Pius A, Thomas S (2017) Chitin nanowhisker (ChNW)-functionalized electrospun PVDF membrane for enhanced removal of Indigo carmine. Carbohyd Polym 165:115-122

Herrera N, Mathew A, Wang L, Oksman K (2011) Randomly oriented and aligned cellulose fibres reinforced with cellulose nanowhiskers, prepared by electrospinning. Plast Rubber Compos 40:57-64

Huang Z, Zhang Y, Kotaki M, Ramakrishna S (2003) A review on polymer nanofibers by electrospinning and their 
applications in nanocomposites. Compos Sci Technol 63:2223-2253

Huang L, Manickam SS, McCutcheon JR (2013) Increasing strength of electrospun nanofiber membranes for water filtration using solvent vapor. J Membr Sci 436:213-220

Inc I (2004) Membrane technology benefits the food processing industry. Filtr Sep 41:32-33

Karim Z, Mathew AP, Grahn M, Mouzon J, Oksman K (2014) Nanoporous membranes with cellulose nanocrystals as functional entity in chitosan: removal of dyes from water. Carbohyd Polym 112:668-676

Karim Z, Mathew AP, Kokol V, Wei J, Grahn M (2016) Highflux affinity membranes based on cellulose nanocomposites for removal of heavy metal ions from industrial effluents. RSC Adv 6:20644-20653

Kaur S, Ma Z, Gopal R, Singh G, Ramakrishna S, Matsuura T (2007) Plasma-induced graft copolymerization of poly (methacrylic acid) on electrospun poly (vinylidene fluoride) nanofiber membrane. Langmuir 23:13085-13092

Li D, Neumann A (1992) Equation of state for interfacial tensions of solid-liquid systems. Adv Coll Interface Sci 39:299-345

Lipnizki F (2010) Cross-flow membrane applications in the food industry. In: Peinemann KV, Pereira S, Giorno L (eds) Membranes for food applications. Willey, Weinheim, pp 1-23

Liu P, Borrell PF, Božič M, Kokol V, Oksman K, Mathew AP (2015) Nanocelluloses and their phosphorylated derivatives for selective adsorption of $\mathrm{Ag}+, \mathrm{Cu} 2+$ and $\mathrm{Fe} 3+$ from industrial effluents. J Hazard Mater 294:177-185

Luo M, Zhao J, Tang W, Pu C (2005) Hydrophilic modification of poly (ether sulfone) ultrafiltration membrane surface by self-assembly of $\mathrm{TiO} 2$ nanoparticles. Appl Surf Sci 249:76-84

Ma Z, Kotaki M, Ramakrishna S (2005) Electrospun cellulose nanofiber as affinity membrane. J Membr Sci 265:115-123
Ma H, Burger C, Hsiao BS, Chu B (2011a) Nanofibrous microfiltration membrane based on cellulose nanowhiskers. Biomacromol 13:180-186

Ma H, Hsiao BS, Chu B (2011b) Ultrafine cellulose nanofibers as efficient adsorbents for removal of UO22 in water. ACS Macro Letters 1:213-216

Manth T, Gabor M, Oklejas E (2003) Minimizing RO energy consumption under variable conditions of operation. Desalination 157:9-21

Mathew AP, Oksman K, Karim Z, Liu P, Khan SA, Naseri N (2014) Process scale up and characterization of wood cellulose nanocrystals hydrolysed using bioethanol pilot plant. Ind Crops Prod 58:212-219

Mautner A, Maples HA, Sehaqui H, Zimmermann T, de Larraya UP, Mathew AP, Lai CY, Li K, Bismarck A (2016) Nitrate removal from water using a nanopaper ion-exchanger. Environ Sci Water Res Technol 2:117-124

Mohammad AW, Ng CY, Lim YP, Ng GH (2012) Ultrafiltration in food processing industry: review on application, membrane fouling, and fouling control. Food Bioprocess Technol 5:1143-1156

Moy E, Neumann A (1987) Solid/liquid interfacial tensions from contact angle data and direct force measurements. J Colloid Interface Sci 119:296-297

Qin A, Li X, Zhao X, Liu D, He C (2015) Preparation and characterization of nano-chitin whisker reinforced PVDF membrane with excellent antifouling property. J Membr Sci 480:1-10

Sehaqui H, de Larraya UP, Tingaut P, Zimmermann T (2015) Humic acid adsorption onto cationic cellulose nanofibers for bioinspired removal of copper (ii) and a positively charged dye. Soft Matter 11:5294-5300

Shahmansouri A, Bellona C (2015) Nanofiltration technology in water treatment and reuse: applications and costs. Water Sci Technol 71:309-319

Shannon MA, Bohn PW, Elimelech M, Georgiadis JG, Marinas BJ, Mayes AM (2008) Science and technology for water purification in the coming decades. Nature 452:301-310 